Original Research Paper

\title{
Studying Interrelation of PDLIM4 with the Model of CD74-Mediated Development of Breast Cancer
}

\author{
${ }^{1,2}$ Dmitry Sergeevich Kravchenko, ${ }^{1,2}$ Yuriy Nikolaevich Lezhnin, \\ ${ }^{1}$ Anna Evgenyevna Ivanova, ${ }^{1}$ Yulia Evgenyevna Kravchenko and ${ }^{1}$ Elena Ivanovna Frolova \\ ${ }^{I}$ Shemyakin-Ovchinnikov Institute of Bioorganic Chemistry of the Russian Academy of Sciences 16/10, \\ Miklukho-Maklaya St., GSP-7, Moscow, 117997, Russian Federation \\ ${ }^{2}$ Engelhardt Institute of Molecular Biology, Russian Academy of Sciences 119991 Moscow, Russia
}

Article history

Received: 16-02-2017

Revised: $10-05-2017$

Accepted: 02-06-2017

Corresponding Author:

Elena Ivanovna Frolova

Shemyakin-Ovchinnikov

Institute of Bioorganic

Chemistry of the Russian

Academy of Sciences 16/10,

Miklukho-Maklaya St., GSP-7,

Moscow, 117997, Russian

Federation

Email: eifr@ibch.ru

\begin{abstract}
Mammary gland tumors are often characterized by deregulation of RIL/PDLIM4 expression, however, PDLIM4-mediated mechanisms of cancer development still remain unknown. The aim of our research was to study the influence of PDLIM4-deregulation on transcript me changes of malignized cells. We performed a comparative NGS-analysis of breast cancer cell lines with suppressed and induced expression of PDLIM4 and identified a number of genes, whose expression correlated with PDLIM4 level. Among them we found CD74 gene, which is known as a potential marker of triple negative subtype of breast cancer. The obtained results allowed us to propose an interrelation between PDLIM4 and the CD74-mediated mechanism of breast cancer development and to analyze the correlation of PDLIM4 with the main components of CD-74-dependent model.
\end{abstract}

Keywords: Breast Cancer, PDLIM4-Mediated Mechanisms, Malignized Cells

\section{Introduction}

Development of breast cancer is a multi-stage process, involving gradual accumulation of a number of genetic and epigenetic disorders, which finally results in anaplasia and formation of cells with high proliferative activity and the potential to invade or spread to other parts of the body (Stratton et al., 2009). Significant reorganizations in the phenotype, metabolism and the key intracellular processes that occur during cancer transformation are controlled by several signaling cascades that lead to considerable changes of the cell transcriptome due to suppression or over expression of numerous target proteins (Engin et al., 2016; Zhu et al., 2016). The constantly increasing amount of data shows that development of different types of tumors is characterized by an alternative signaling cascades that change the cell transcription profile in various ways (Inic et al., 2014; Lin et al., 2016). Therefore, identification of interactions between individual intracellular proteins in healthy and cancer cells brings us closer to composing of complete picture of cell proteome and contributes to revealing of development algorithms in various types of tumors.

The RIL gene (reversion-induced LIM-domain), which was later named PDLIM4, was first described in
1995 as a potential oncology suppressor (Kiess et al., 1995). Since then, a lot of supporting evidence has been collected that expression of this protein is frequently deregulated at genetic and epigenetic levels in tumors of various types (Bashirova et al., 1998; Vanaja et al., 2006; Boumber et al., 2007; Feng et al., 2010; Xu et al., 2012). PDLIM4 can play a special role in development of Breast Cancer (BC): Some data indirectly indicates correlation of certain clinical parameters of tumor: Size, ploidy and depth of cell differentiation with PDLIM4 overexpression or suppression (Xu et al., 2012). Despite available evidence of correlation between PDLIM4 expression levels and clinical characteristics of different types of cancer, the mechanisms of tumor development in which PDLIM4 takes part, remain unknown.

Previous studies of PDLIM4 expression showed that different breast cancer tumors are characterized by high polymorphism in the intracellular level of this protein Neve, Chin et al. (2004). Even within separate subtypes of $\mathrm{BC}$, it is possible to distinguish the lines that are PDLIM4positive and PDLIM4-negative (Kravchenko et al., 2015; Lawrence et al., 2015). These results allow to assume the existence of an alternative molecular mechanisms of tumor development, which are associated with suppression or overexpression of PDLIM4, which indicates PDLIM4's ability to act both as an oncosuppressor and an oncogene, depending on the type 
of the tumor. Previously we focused on studying BC cell lines with elevated levels of PDLIM4 expression and assessing the influence of PDLIM4 silencing on the changes in the cell transcriptome (Kravchenko et al., 2016). To obtain a complete picture of PDLIM4 involvement in the intracellular signaling, it seemed necessary to additionally analyze BC cell lines with suppressed expression of PDLIM4 and to assess contribution of PDLIM4 overexpression in the changes in the transcriptome of breast cancer cell lines.

\section{Materials and Methods}

\section{Cell Culture}

The brief characteristic of the cell cultures used in the work is shown in Table 1. Cells were cultivated in the Eagle medium modified by Dyulbekko (DMEM) with added $2 \mathrm{mmol}$ of L-glutamine, $4.5 \mathrm{~g} \mathrm{~L}^{-1}$ of glucose, 100 Units $\mathrm{mL}^{-1}$ of penicillin and $100 \mathrm{ug} \mathrm{mL} \mathrm{m}^{-1}$ of streptomycin with addition of $10 \%$ of Embryonic Veal Serum (EVS) (BioWhitacker). Cultivation was performed at $37^{\circ} \mathrm{C}, 5 \%$ of $\mathrm{CO}_{2}$.

\section{Transfection}

The cells were seeded $24 \mathrm{~h}$ before the transfection, at the time of transfection their density did not exceed $70 \%$ of the monolayer. The transfection was performed using the liposome method with the use of LipofectAmine and Plus-reagents (Invitrogen) according to the manufacturer's protocol. The transfection-grade DNA and Plus-reagent were dissolved in a serum-free medium with the recommended ratios. The mixture was incubated for $15 \mathrm{~min}$ at room temperature and diluted LipofectAmine was added to this mixture, which was then left to incubate for another $15 \mathrm{~min}$ for liposomes formation. Meanwhile, the culture medium for the cells was replaced with a serum-free one. The obtained DNA-liposome complexes were added to the cells and incubated during 3 to $16 \mathrm{~h}$, depending on the cell line and then medium was replaced with DMEM containing $10 \%$ FCS. For protein over-expression constructs, the cells were incubated from the moment of transfection for not less than $20 \mathrm{~h}$ and for RNA-interference induction and maximum suppression of mRNA target, shRNA was expressed for not less than $48 \mathrm{~h}$.

\section{Packing Lentiviral Particles}

To obtain lentivirus particles, cell line HEK293T was used, which is characterized by very high expression of proteins from exogenous constructs. Cells were seeded in the ratio of 1:4 $16 \mathrm{~h}$ before the transfection, at the time of transfection their density was about $70 \%$ of the monolayer. The transfection was performed as described above. Co-transfection of 3 or 4 plasmids: The lentivirus structure itself that codes the virus RNA genome; one
(pCMV $\Delta$ R8.2) or two (pGag1 and pRev2) helper plasmid that encode viral structural proteins, reverse transcriptase and proteases; virions were pseudotyped by t protein G of the Vesicular Stomatitis Virus (pVSV-G), which does not require cellular receptors for cell entry, thus ensuring high efficiency of transduction and a wide range of susceptible cells. The medium of transfected cells containing recombinant virions was collected after 24, 36, 48 and $72 \mathrm{~h}$, filtered through $0.44 \mu \mathrm{m}$ sterile filters (Millipore) and used for infecting target cells. The virus stocks were stored frozen at $-70^{\circ} \mathrm{C}$. To decrease the concentration of toxic cellular metabolites in virus stocks, a medium with a lower content of serum was used if necessary ( 5 or $2.5 \%$ of fetal calf serum).

\section{Cleaning of Virions and Transduction}

For infecting target cells, they were specially seeded 16-24 $\mathrm{h}$ before treatment in order to get density equal to $50-70 \%$ of the monolayer. The virus stocks were diluted with a rich medium, the polybrene was added in the concentration of 4-8 ug $\mathrm{mL}^{-1}$ to increase infection efficiency and the virus stock was added to the cells. Infection was performed in the minimum volume of the medium for 4-8 h. To increase the probability of transduction of poorly infected cells, incubation was performed with the use of a rotational shaker (20-30 rpm).

To decrease toxicity of the virus stocks and to increase virus particles titer, they were concentrated by precipitation with PEG-8000, when necessary. $1 / 3$ of the volume of sterile $40 \%$ PEG- 8000 solution prepared in PBS was added to the fresh-gathered virus-containing supernatants, then they were mixed and incubated on ice for not less than $8 \mathrm{~h}$ (in this condition, virus particles are stable for at least 7 days). The interconnected virions were gathered by centrifugation at $+4^{\circ} \mathrm{C}, 8000 \mathrm{~g}, 15$ min., the supernatant was carefully removed and the deposit was resuspended in PBS or in a rich growth medium and the aliquots were stored frozen at $-70^{\circ} \mathrm{C}$.

\section{The Genetic Constructs used in the Work}

PDLIM4 fused with the Flag epitope was expressed by the PLM-CMV-FlagRIL vector that ensures high level of constitutive protein expression under the control of the cytomegalovirus promotor.

\section{$R T-P C R$}

For obtaining cDNA, reverse transcription was performed using reverse transcriptase SuperScript II RT (Invitrogen) with the use of oligo (dT)12-18 primer on 2 ug of total RNA. The method of reverse transcription is described in the instruction to the set of reagents SuperScript First-strand Synthesis System for RT-PCR (Invitrogen). The aliquots were added to the standard mix for PCR-amplification $(25 \mu \mathrm{L})$ in portions of $2 \mu \mathrm{L}$ of cDNA. 
Table 1. The cellular cultures used in the work and their source

\begin{tabular}{ll}
\hline Cell line & Origin \\
\hline 293T & Human embryonic kidney cells, transformed DNA from the early area of adenovirus of the 5-th type \\
MCF7 & Adenocarcinoma of a human mammary gland, metastatic effusion of pleura (ATCC number HTB-22) \\
BT-20 & Carcinoma of a human mammary gland (ATCC number HTB-19) \\
T-47D & Infiltrative ductal carcinoma of a human mammary gland, metastatic pleural effusion (ATCC number HTB-133) \\
MDA-MB-468 & Adenocarcinoma of a human mammary gland, metastatic pleural effusion (ATCC number HTB-132) \\
MDA-MB-231 & Adenocarcinoma of a human mammary gland, pleural effusion (ATCC number HTB-26) \\
MDA-MB-435S & Ductal carcinoma of a human mammary gland, metastatic pleural effusion (ATCC number HTB-129) \\
MDA-MB-436 & Adenocarcinoma of a human mammary gland, metastatic pleural effusion (ATCC number of HTB- 30) \\
BT-474 & Ductal carcinoma of a human mammary gland, (ATCC number HTB-20) \\
\hline
\end{tabular}

For PDLIM4 amplification, the following sequences of primers were used:

\section{PDLIM4 direct: 5'-CTCGCTTTCCAGTCCCTCACAAT PDLIM4 reverse: 5'-TCTAGCATGCCCTGCAAGTAGC}

12.5 pmol of each primer were used. The temperature of primers annealing was calculated in the OligoAnalyser application that is available at the Internet page of the IDT DNA (www.idtdna.com/scitools). To monitor the efficiency of reverse transcription and alignment, sections of mRNA of «household» genes were amplified: RP-2 and GAPDH.

\section{RNA Separation, Quality Assessment, Hybridization on Microchips}

The total RNA was separated with the use of Trizol reagent, according to the protocol recommended by the manufacturer (Invitrogen). In course of the process, 200 $\mu \mathrm{L}$ of chloroform were added to samples containing 1 $\mathrm{mL}$ of a lysate and carefully mixed. The obtained suspension was centrifuged at $12,000 \mathrm{~g}$ for $15 \mathrm{~min}$ at $4^{\circ} \mathrm{C}$. The top part of solution was transferred to clean test tubes and $500 \mu \mathrm{L}$ of isopropanol were added. Samples were incubated on ice for an hour, then they were centrifuged for $10 \mathrm{~min}$., $12,000 \mathrm{~g}$, , at $4^{\circ} \mathrm{C}$, then isopropanol was removed. The RNA precipitate was washed with $75 \%$ solution of ethanol, which was removed by further centrifugation (10 min., 12,000 g, $4^{\circ} \mathrm{C}$ ). Next, the RNA pellet was dried for 5-10 min at room temperature and resuspended in $50 \mu \mathrm{L}$ of water, cleaned from RNAses and processed with DNAse I (Promega). Concentration of the obtained RNA was measured using a NanoDrop ND-1000 spectrophotometer (Thermo Scientific, USA); RNA integrity was checked by denaturating electrophoresis in agarose gel and confirmed during capillary electrophoresis (Agilent Bioanalyzer, Palo Alto, CA). At the first stage of sample preparation, ribosomal RNA was removed by RNA Minus Transcriptome Isolation Kit (Invitrogen). The cleaned samples of total RNA were fragmented in the presence of bivalent cations at high temperature. After that, the obtained RNA fragments were used as a matrix for cDNA synthesis with the use of oligo-dT18 primer and reverse transcriptase. The complementary chain of DNA was further synthesized using DNA polymerase I and RNAse H. Two-chained fragments of cDNA were further ligated with adapters and were amplified in course of PCR for obtaining the complete cDNA library. cDNA was sequenced with the use of Illumina HiSeq 2000 System (The Moscow State University n.a. M. V. Lomonosov). The differential analysis of the obtained data was performed by means of CLC Genomic Workbench 6.0.5. Database for Annotation, Visualization and Integrated Discovery $\begin{array}{llll}\text { (DAVID) } & \text { version } & 6.7 & \text { software }\end{array}$ (http://david.abcc.ncifcrf.gov/home.jsp).

\section{Immunostaining and Cell Analysis}

For immune fluorescent staining, the cells were fixed with 3.7\% formaldehyde solution in PBS (15 min., $25^{\circ} \mathrm{C}$ ), washed with PBS three times (10 min. each). Fixed cells were permeabilized with a $0.5 \%$ Triton $\mathrm{X}$ 100 solution (Sigma, USA) in PBS $\left(7 \mathrm{~min} ., 25^{\circ} \mathrm{C}\right)$, washed with PBS (3 times $10 \mathrm{~min}$. each). Cells were incubated in a $3 \%$ solution of BSA in PBS (40 min., $\left.25^{\circ} \mathrm{C}\right)$. They were incubated with solutions of primary $\left(40\right.$ min., $\left.25^{\circ} \mathrm{C}\right)$ and then secondary antibodies (120 min., $\left.25^{\circ} \mathrm{C}\right)$; immediately after incubation, each solutions was washed with PBS (3 times $10 \mathrm{~min}$. each). The cover glasses were placed on coverslips and Fluoromount Aqueous Mounting Medium (Sigma, the USA) was used for the filling medium.

The obtained preparations were analyzed by means of fluorescent microscope Zeiss Axiovert 200 M (Carl Zeiss Micro Imaging, Germany) with an Axio Cam Hrm camera. The obtained images were processed in Image J application.

\section{Results}

Among $\mathrm{BC}$ lines that are characterized by suppressed expression of PDLIM4, we chose T-47D line that represents a sample of an infiltrative ductal carcinoma of human mammary gland. The chosen cells were successfully transfected by the PLM-CMV-FLAG-RIL vector, which ensures high level of PDLIM4 expression. A significant increase of PDLIM4 level was confirmed by PCR, the results are shown in Fig. 1 and 3A 
In order to detect epigenetic changes that occurred in the cells due to the change of PDLIM4 expression level, mRNA was isolated from the cells of initial and transgene lines, which was later used for analyzing transcriptome in NGS-sequencing. Quantitative data was obtained about expression of a wide selection of genes for the cells in both BC lines. Due to the fact that the difference in expression profiles between the studied lines was directly related to the change of PDLIM4 expression level, the performed analysis allowed detecting the genes, whose products directly interact with PDLIM4, or participate in adjacent intracellular processes. The detected genes were divided into two groups: the first group included the genes in which expression was upregulated upon increasing level of PDLIM4, the second included the genes which expression was suppressed at the same time. It seemed appropriate to compare the obtained results with those of previous NGS transcriptomes sequencing, the purpose of which was identification of the genes associated with suppressing PDLIM4 expression (Kravchenko et al., 2016). Performing two consecutive sequencings after suppression and activation of PDLIM4 expression in cells of various lines of $\mathrm{BC}$ allowed us to compare the obtained results and to detect the genes, which expression correlated with PDLIM4 expression in both experiments. The genes, whose expression equally correlates with PDLIM4 expression, in case of suppression and in case of increasing its expression, are shown in Table 2.

Among the obtained results, the most interesting was the direct correlation between PDLIM4 expression and CD74 expression level. CD74 is a $\gamma-$ subunit of the Main II class Histocompatibility Complex (MHC), hyper expression of which is often observed in malignant tumors of epithelial and mesenchymal nature (Borghese and Clanchy, 2011; Otterstrom et al., 2014). The increased expression of CD74 is associated with active metastasis and negative forecast of breast tumors development (Leth-Larsen et al., 2010; Greenwood et al., 2012; Tian et al., 2012). The previous data about studying correlation of violation of PDLIM4 expression in cells of the tumors that belong to various subtypes of $\mathrm{BC}$ indicates that hyperexpression of PDLIM4 is most characteristic for cells of claudindeficient subtype of the triple negative $\mathrm{BC}$ type (Kravchenko et al., 2015). According to this data, it seems rather interesting to assess direct correlation between PDLIM4 expression and CD74, which was also offered as a diagnostic marker of triple negative $\mathrm{BC}$ type (Tian et al., 2012). One can assume that both proteins take part in the adjacent intracellular process, which is associated with development of this type of cancer. Mechanisms of CD74-mediated BC formation have been poorly studied; the only existing model today is the concept of Scribble-dependent formation of tumors. According to this model, an increased amount of intracellular CD74 influences functional Scribble activity, the product of the well-known tumor suppressor Scrib, which is crucial for maintaining polarity of epithelial cells (Qin et al., 2005; Metodieva et al., 2013). In humans, Scribble is required for maintaining Ecadherin-dependent intercellular interactions: suppression of Scribble expression, or loss of its activity results in decreased expression of E-cadherin, violation of adhesive contacts and acquisition of the mesenchymal phenotype by the cells, related to high migratory activity and invasiveness (Qin et al., 2005; Su et al., 2012). It is interesting that suppression of Scribble expression is often observed especially in case of breast cancer studied in our work (Zhan et al., 2008).

Generalizing the provided data, one can assume that the increased PDLIM4 expression, by directly correlating with CD74 expression, will also be associated with inactivation of Scribble and with suppression of E-cadherin expression, which, as a result, will stimulate epithelial-mesenchymal transformation of mammary gland epithelium cells and will lead to cancer development, as shown in Fig. 2.

In order to check this assumption, it was necessary to analyze the correlation of PDLIM4 expression level with each of components of the described model. In the first stage of work, during the immune-enzyme and immune-fluorescent analysis, direct correlation of level of PDLIM4 and CD74 expression (Fig. 3A and 4A) was confirmed, which had been traced before only by expression by mRNA. Analyzing the micro photo in Fig. 4A, one can see that cells that most intensively express PDLIM4 are characterized by higher CD74 expression; moreover, these proteins tend to colocalize. Similar regularity in the level of PDLIM4 and CD74 expression can be seen in Fig. 3A.

Table 2. List of genes, whose expression equally correlates with PDLIM4 expression, both in case of suppression and in case of increasing of PDLIM4 level in cells of MDAMB-231 and T-47D lines, respectively

Genes correlating with PDLIM4 expression level

\begin{tabular}{ll} 
Direct correlation & Inverse correlation \\
\hline CD74 & ALDH3A2 \\
HLA-DRA & \\
ICAM1 & \\
c3 & \\
\hline
\end{tabular}




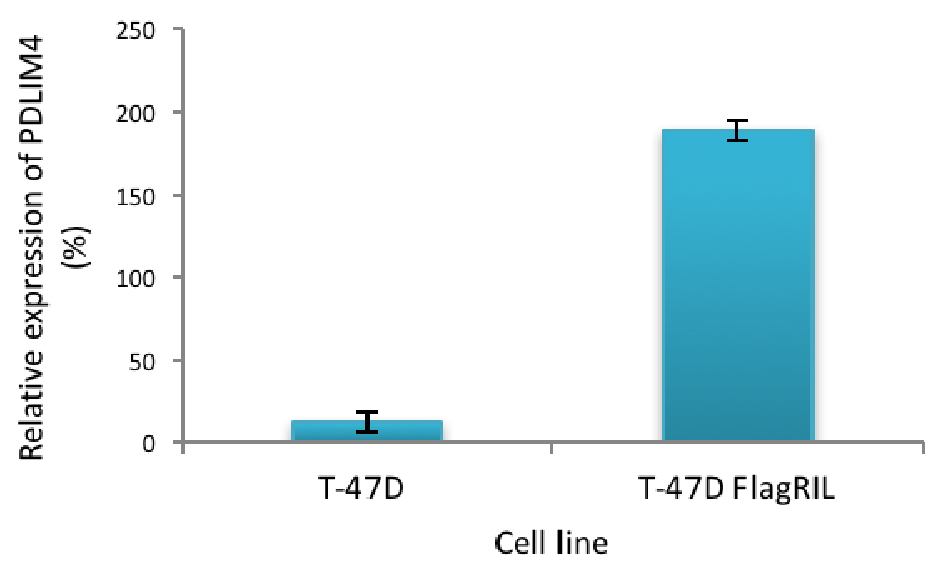

Fig. 1. Results of the PCR-analysis of PDLIM4 expression level in cells of the initial and transgene T-47D lines; data represent mean $\pm \mathrm{SE}(\mathrm{n}=3) .{ }^{*}$-value $<0.01(\mathrm{t}$-test $)$

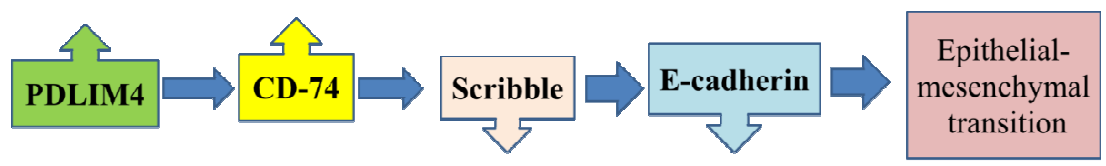

Fig. 2. The hypothetical scheme of PDLIM4 interrelation with CD74-mediated mechanism of BC development

A

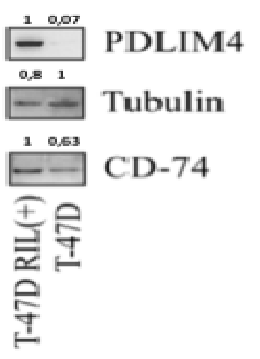

B

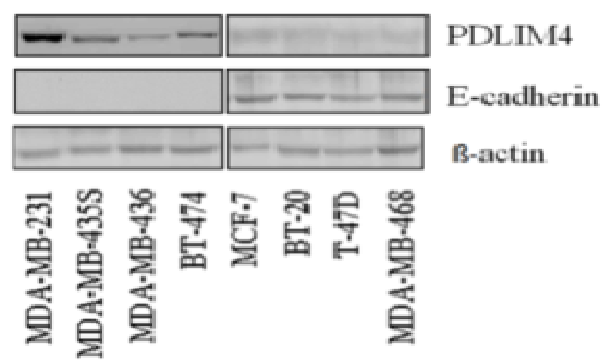

Fig. 3. Results of Western-blot analysis of PDLIM4 expression correlation with expression intensity: A- CD74 in cells of the initial and transgene T-47D lines; densitometry was performed and the relative level of each protein is displayed below each lane; B - E-cadherin in BC lines. A-tubulin and B-beta-actin were used for reference

Identification of the interrelation between PDLIM4 and Scribble became the next stage of the study. According to a number of researches, an increased CD74 level in cells does not result in considerable decrease of Scribble expression; however, it causes inactivation of this protein (Metodieva et al., 2013). During Scribble interaction with CD74, the status of phosphorylation of the specific sites of the C-terminal site of Scribble changes, which leads to decreased functional activity. The active Scribble form is normally located on a basallateral membrane that is required for its ability to inhibit G1/S transition. However, in course of CD74-dependent Scribble inactivation, dephosphorylated protein moves from sections of intercellular contacts to the cytoplasm, which further leads to acquisition of mesenchymal phenotype by the cells (Metodieva et al., 2013). Thus, for analyzing the interrelation of the level of PDLIM4 expression with Scribble activity, it was possible to trace the intracellular localization of Scribble in PDLIM4 (+) and PDLIM4 (-) cells. During the work, we used initial and transgene T-47D lines. The results of the experiment are shown in Fig. 4B.

Analyzing the presented micro-photos, one can notice that in PDLIM4-deficient cells, Scribble is mainly located on the periphery, in the locations of intercellular contacts. This nature of intracellular localization allows to make a conclusion about the functional activity of the protein: The close-to-membrane localization is characteristic of the phosphorylated form of Scribble and is required for its normal functioning (Metodieva et al., 2013). At the same time, in the cells characterized by high level of PDLIM4 expression, one can see delocalization of Scribble from the locations of intercellular contacts, which indicates the loss of functional activity of the protein. This data shows that high level of PDLIM4 expression can be associated with Scribble inactivation, which is consistent with our conception of PDLIM4-dependent process of $\mathrm{BC}$ formation. 

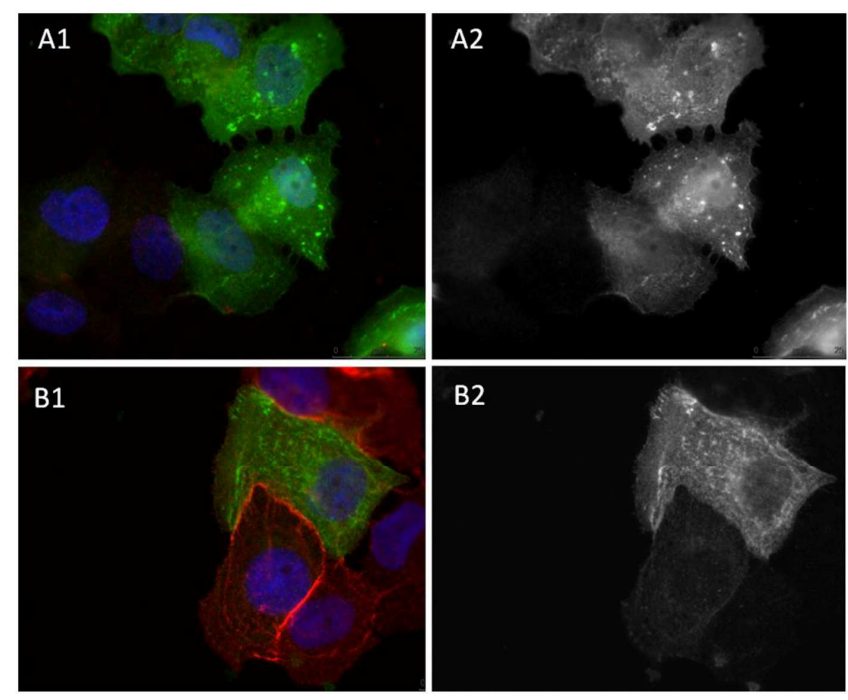
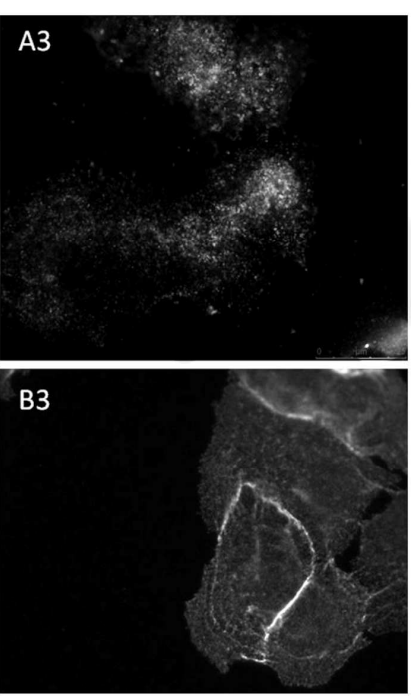

Fig. 4. Results of T-47D Flag RIL cells immune fluorescent staining, (A) analysis of PDLIM4 and CD74 expression: (A1) PDLIM4 (stained by green) and CD74 (stained by red), (A2) anti-PDLIM4 staining, (A3) anti-CD74 staining; (B) analysis of PDLIM4 and Scribble expression: (B1) PDLIM4; (stained by green) and Scribble (stained by red), (B2) anti-PDLIM4 staining, (B3) anti-Scribble staining; the nuclei are stained by DAPI (blue)

The final stage of our study was the assessment of the correlation between PDLIM4 expression and E-cadherin - the key link of the proposed model. The loss of this link leads to epithelial-mesenchymal transition and to cancer development. During the work, the results of immune-enzyme analysis of E-cadherin expression was analyzed in cells of some BC lines with PDLIM4 protein expression; the obtained results are shown in Fig. 3B. It is possible to see that in each of the eight $\mathrm{BC}$ lines that were used in the work, the reverse correlation of expression of the studied proteins is found: high level of E-cadherin expression turns out to be characteristic of PDLIM4-deficit lines, while in cells of PDLIM4-positive lines, expression of E-cadherin turns out to be suppressed. This is also completely in line with the proposed mechanism of PDLIM4 interrelation with the model of CD74 - mediated development of BC.

\section{Discussion}

The results obtained during the work show tight connection between disrupted expression of PDLIM4 and CD74-mediated mechanism of breast cancer development. Within the described model, PDLIM4 shows oncogenic properties and turns out to be associated with the most negative cancer forecast. The provided data makes it possible to explain why for several most malignant lines of BC, such as MDA-MB-231, MDA-MB-438 or HCC38, the high level of PDLIM4 expressionism is characteristic (Lawrence et al., 2015).

Despite of the established correlation of endogenous PDLIM4 quantity with the level of CD74, Scribble and E-cadherin expression, the mechanisms of regulation and possible intracellular interaction of these proteins remain unclear. Based on the previous research data it is possible to assume that described proteins are components of the same signaling cascades involved in $\mathrm{BC}$ developments. It is known, in particular, that CD74, acting as a cellular receptor for MIF-factor that inhibits migration of macrophages can activate ERK signaling path (Ras-ERK, MAPK/ERK), one of the key and most well studied signaling paths of MAPK (mutagen-activated protein kinase) (Fan et al., 2011; Yamben et al., 2013). The previous results of studying the role of PDLIM4 in BC development also showed its interrelation with separate components of a MAPK signaling path (Kravchenko et al., 2016). Paying attention to the discovered tendency to PDLIM4 and CD74 colocalization (Fig. 4A), it is also possible that these proteins directly interact with each other. Studying the possible mechanisms of implementation of the discovered interrelation between PDLIM4 and CD74, Scribble and E-cadherin might become subject of further research and will let unambiguously assess the role of PDLIM4 in breast cancer formation.

\section{Conclusion}

As a result of analyzing the deep sequencing data of transcriptomes of cell lines of a mammary gland with activated or suppressed PDLIM4 expression, we propose a new mechanism of cell malignization. Apparently, high levels of PDLIM4 expression may have a stimulating impact on CD74 expression, which may lead to suppression of the activity of intracellular tumor suppressor, Scribble and suppress E-cadherin expression, which, in turn, may promote epithelial-mesenchymal transition and increase malignancy of tumor cells. 


\section{Funding Information}

The work funded by MESR contract №14.607.21.0062 (RFMEFI60714X0062).

\section{Author's Contributions}

Dmitry Sergeevich Kravchenko: Immunostaining, RT-PCR, cell culture.

Yuriy Nikolaevich Lezhnin: Plasmid construction, lentiviral transduction.

Anna Evgenyevna Ivanova: Western-blotting.

Yulia Evgenyevna Kravchenko: RNA preparation, purification and QC.

Elena Ivanovna Frolova: Research planning and supervision.

\section{Ethics}

This research has not been submitted for publication nor has it been published in whole or in part elsewhere. We attest to the fact that all Authors listed on the title page have contributed significantly to the work, have read the manuscript, attest to the validity and legitimacy of the data and its interpretation and agree to its submission.

\section{Reference}

Bashirova, A.A., M.L. Markelov, T.V. Shlykova, E.V. Levshenkova and Alibaeva RA et al., 1998. The human RIL gene: Mapping to human chromosome 5q31.1, genomic organization and alternative transcripts. Gene, 210: 239-245.

Borghese, F. and F.I. Clanchy, 2011. CD74: An emerging opportunity as a therapeutic target in cancer and autoimmune disease. Expert Opin. Ther. Targets, 15: 237-251.

Boumber, Y.A., Y. Kondo, X. Chen, L. Shen and V. Gharibyan et al., 2007. RIL, a LIM gene on 5q31, is silenced by methylation in cancer and sensitizes cancer cells to apoptosis. Cancer Res., 67: 1997-2005. DOI: 10.1158/0008-5472.CAN-06-3093

Chin, K., C.O. de Solorzano, D. Knowles, A. Jones and W. Chou et al., 2004. In situ analyses of genome instability in breast cancer. Nat. Genet. 36: 984-988

Engin, H.B., J.F. Kreisberg and H. Carter, 2016. Structure-based analysis reveals cancer missense mutations target protein interaction interfaces. PLoS One. DOI: 10.1371/journal.pone.0152929

Fan, H., P. Hall, L.L. Santos, J.L. Gregory and G. Fingerle-Rowson et al. 2011. Macrophage migration inhibitory factor and CD74 regulate macrophage chemotactic responses via MAPK and Rho GTPase. J. Immunol., 186: 4915-4924. DOI: $10.4049 /$ jimmunol.1003713

Feng, W., R. Orlandi, N. Zhao, M.L. Carcangiu and E. Tagliabue et al., 2010. Tumor suppressor genes are frequently methylated in lymph node metastases of breast cancers. BMC Cancer, 10: 378-378. DOI: $10.1186 / 1471-2407-10-378$

Greenwood, C., G. Metodieva, K. Al-Janabi, B. Lausen and L. Alldridge et al., 2012. Tat1 and CD74 overexpression is co-dependent and linked to increased invasion and lymph node metastasis in triplenegative breast cancer. J. Proteom., 75: 3031-3040. DOI: $10.1016 /$ j.jprot.2011.11.033

Inic, Z., M. Zegarac, I. Momcilo, I. Markovic and Z. Kozomara et al., 2014. Difference between luminal $\mathrm{a}$ and luminal $\mathrm{b}$ subtypes according to ki67 , tumor size and progesterone receptor negativity providing prognostic information. Clin. Med. Insights Oncol., 8: 107-111. DOI: 10.4137/CMO.S18006

Kiess, M., B. Scharm, A. Aguzzi, A. Hajnal and R. Klemenz et al., 1995. Expression of ril, a novel LIM domain gene, is down-regulated in Hrastransformed cells and restored in phenotypic revertants. Oncogene, 10: 61-68.

Kravchenko, D.S., Y.N. Lezhnin, J.E. Kravchenko, S.P. Chumakov and E.I. Frolova, 2016. Study of molecular mechanisms of PDLIM4/RIL in promotion of the development of breast cancer. Biol. Med., 8: 2-2.

Kravchenko, D.S., Y.N. Lezhnin and E.I. Frolova, 2015. Analysis of PDLIM4 expression in different subtypes of breast cancer. Biosci. Biotechnol. Res. DOI: $10.13005 / \mathrm{bbra} / 1866$

Lawrence, R.T., E.M. Perez, D. Hernández, C.P. Miller and K.M. Haas et al., 2015. The proteomic landscape of triple-negative breast cancer. Cell Rep., 11: 630-644. DOI: 10.1016/j.celrep.2015.03.050

Leth-Larsen, R., R.R. Lund and H.J. Ditzel, 2010. Plasma membrane proteomics and its application in clinical cancer biomarker discovery. Mol Cell Proteom., 9: 1369-1382. DOI: $10.1074 / \mathrm{mcp} . \mathrm{R} 900006-\mathrm{MCP} 200$

Lin, A., C. Li, Z. Xing, Q. Hu and K. Liang et al., 2016. The link-a lncRNA activates normoxic HIF1 $\alpha$ signalling in triple-negative breast cancer. Nat. Cell Biol., 18: 213-224. DOI: 10.1038/ncb3295

Metodieva, G., N.C. Nogueira-de-Souza, C. Greenwood, K. Al-Janab and L. Leng et al., 2013. CD74dependent deregulation of the tumor suppressor scribble in human epithelial and breast cancer cells. Neoplasia, 15: 660-668. DOI: $10.1593 /$ neo. 13464

Otterstrom, C., A. Soltermann, I. Opitz, E. Felley-Bosco and W. Weder et al., 2014. CD74: A new prognostic factor for patients with malignant pleural mesothelioma. Br. J. Cancer, 110: 2040-2046. DOI: $10.1038 /$ bjc.2014.117

Qin, Y., C. Capaldo, M. Gumbiner and I.G. Macara, 2005. The mammalian scribble polarity protein regulates epithelial cell adhesion and migration through E-cadherin. J. Cell Biol., 171: 1061-1071. DOI: $10.1083 /$ jcb.200506094 
Stratton, M.R., P.J. Campbell and P.A. Futreal, 2009. The cancer genome. Nature, 458: 719-724. DOI: 10.1038 /nature 07943

Su, W.H., D.D. Mruk, E.W. Wong, W.Y. Lui and C.Y. Cheng, 2012. Polarity protein complex scribble/Lgl/Dlg and epithelial cell barriers. Adv. Exp. Med. Biol., 763: 149-170.

Tian, B., Y. Zhang, N. Li, L. Xuewen and J. Dong, 2012. CD74: A potential novel target for triple-negative breast cancer. Tumour Biol., 33: 2273-2277. DOI: $10.1007 / \mathrm{s} 13277-012-0489-\mathrm{x}$

Vanaja, D.K., K.V. Ballman, B.W. Morlan, J.C. Cheville and R.M Neumann, 2006. PDLIM4 repression by hypermethylation as a potential biomarker for prostate cancer. Clin. Cancer Res., 12: 1128-1136. DOI: 10.1158/1078-0432.CCR-05-2072

$\mathrm{Xu}$, J., P.B. Shetty, W. Feng, C. Chenault and R.C. Bast, et al., 2012. Methylation of HIN-1, RASSF1A, RIL and CDH13 in breast cancer is associated with clinical characteristics, but only RASSF1A methylation is associated with outcome. BMC Cancer. DOI: 10.1186/1471-2407-12-243
Yamben, I.F., R.A. Rachel, S. Shatadala, N.G. Copeland and N.A. Jenkins et al., 2013. Scrib is required for epithelial cell identity and prevents epithelial to mesenchymal transition in the mouse. Dev. Biol., 384: 41-52. DOI: 10.1016/j.ydbio.2013.09.027

Zhan, L., A. Rosenberg, K.C. Bergami, M. Yu and Z. Xuan et al., 2008. Deregulation of scribble promotes mammary tumorigenesis and reveals a role for cell polarity in carcinoma. Cell, 135: 865-878. DOI: $10.1016 /$ j.cell.2008.09.045

Zhu, P., H.M. Aliabadi, H. Uludağ and J. Han, 2016. Identification of potential drug targets in cancer signaling pathways using stochastic logical models. Sci. Rep. DOI: 10.1038/srep23078 